Management international

International Management

Gestiòn Internacional

\title{
Impacts de l'expatriation en pays à risques sur les hiérarchies organisationnelles et sociales
}

Mobilité sociale d'expatriés de pays développés et en développement au Nigeria

\section{Consequences of hardship posts upon corporate and social hierarchies Social mobility of First and Third World expatriates in Nigeria Consecuencias de la expatriación en países de riesgo en las jerarquías profesionales y sociales \\ Movilidad social de expatriados de países desarrollados y en desarrollo}

François Goxe et Marjolaine Paris

Volume 18, numéro hors-série, 2014

Ancrages culturels et dynamiques du Management International

Cultural and Dynamic Roots of International Management

Anclajes culturales y dinámicos de la Gestión Internacional

URI : https://id.erudit.org/iderudit/1027868ar

DOI : https://doi.org/10.7202/1027868ar

Aller au sommaire du numéro

Éditeur(s)

HEC Montréal

Université Paris Dauphine

ISSN

1206-1697 (imprimé)

1918-9222 (numérique)

Découvrir la revue

Citer cet article

Goxe, F. \& Paris, M. (2014). Impacts de l'expatriation en pays à risques sur les hiérarchies organisationnelles et sociales : mobilité sociale d'expatriés de pays développés et en développement au Nigeria. Management international/ International Management / Gestiòn Internacional, 18, 106-122.

https://doi.org/10.7202/1027868ar

\section{Résumé de l'article}

Cet article montre que les managers expatriés accumulent divers capitaux lors d'une expatriation dans un pays à risques. Cependant, le développement de ces capitaux dépend notamment du pays d'origine des expatriés et de leur statut social avant l'expatriation. Les expatriés issus de pays émergents et des classes moyennes de pays développés voient leurs niveaux de capitaux augmenter plus fortement que les autres proportionnellement à leur dotation de départ, mais sont confrontés à un " plafond de verre " qui limite leur mobilité ascendante au sein de l'entreprise comme de la communauté des « managers globalisés ».
Tous droits réservés @ Management international / International Management / Gestión Internacional, 2014
Ce document est protégé par la loi sur le droit d'auteur. L’utilisation des services d'Érudit (y compris la reproduction) est assujettie à sa politique d'utilisation que vous pouvez consulter en ligne.

https://apropos.erudit.org/fr/usagers/politique-dutilisation/ 


\section{Impacts de l'expatriation en pays à risques sur les hiérarchies organisationnelles et sociales Mobilité sociale d'expatriés de pays développés et en développement au Nigeria}

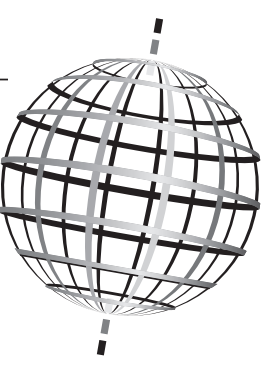

\section{Consequences of hardship posts upon corporate and social hierarchies Social mobility of First and Third World expatriates in Nigeria}

\section{Consecuencias de la expatriación en países de riesgo en las jerarquías profesionales y sociales Movilidad social de expatriados de países desarrollados y en desarrollo}

FRANCOIS GOXE *

Université de Versailles Saint-Quentin-en-Yvelines, France

\author{
MARJOLAINE PARIS* \\ Unité de Recherche Migrations et Société (URMIS) \\ de l'Université Paris Diderot
}

\section{RÉSUMÉ}

Cet article montre que les managers expatriés accumulent divers capitaux lors d'une expatriation dans un pays à risques. Cependant, le développement de ces capitaux dépend notamment du pays d'origine des expatriés et de leur statut social avant l'expatriation. Les expatriés issus de pays émergents et des classes moyennes de pays développés voient leurs niveaux de capitaux augmenter plus fortement que les autres proportionnellement à leur dotation de départ, mais sont confrontés à un «plafond de verre» qui limite leur mobilité ascendante au sein de l'entreprise comme de la communauté des «managers globalisés ».

Mots clés : expatriation, mobilité géographique et sociale, classes, pays émergents

\section{AbSTRACT}

This article shows that expatriate managers do accumulate various forms of capital during expatriation in hardship locations. However, the development of such capital depends in particular on expatriates' country of origin and social status before expatriation. In proportion to their capital endowment before expatriation, expatriates from emerging countries and middleclass managers from developed countries increase their capital more than other expatriates do but they are faced with a "glass ceiling" that limits their upward mobility within the firm and the community of "global managers".

Keywords : expatriation, spatial and social mobility, classes, emerging countries

\section{RESUMEN}

Este artículo demuestra como ejecutivos expatriados acumulan varios capitales durante su expatriación en un país de riesgo. Sin embargo, la acumulación de estos capitales depende en particular de su país y de su estatuto social de origen. Los expatriados de países en desarrollo y de las clases medias de países desarrollados aumentan más sus niveles de capitales que los otros en comparación con su dotación inicial, pero están confrontados a un «techo de cristal» limitando su ascensión social dentro de la empresa y de la comunidad de los ejecutivos internacionales.

Palabras claves : expatriación, movilidad geográfica y social, clases, países en desarrollo a mondialisation des échanges conduit à différentes
formes de mobilité pour les travailleurs qualifiés et non
qualifiés. Une grande part de la littérature en management
international s'est intéressée aux expatriés, le plus souvent
* Remerciements : Les auteurs tiennent à remercier l'éditeur, les
évaluateurs anonymes ainsi que les coordinateurs (J-F. Chanlat, J-P.
Dupuis) et participants (notamment C. Barmeyer et E. Davoine) de
l'atelier Management Interculturel du colloque ATLAS-AFMI 2013 entendus comme des employés de grandes firmes multinationales mutés de façon temporaire à l'étranger avant d'être rapatriés (Cerdin, 2004; Cerdin et Le Pargneux, 2009). L'émergence d'expatriés moins «traditionnels », s'expatriant

pour leurs commentaires et suggestions qui ont contribué à l'élaboration de la version finale de cet article. Nous remercions également O.Vilaça de nous avoir fourni plusieurs de ses articles ainsi que pour ses réflexions. 
par eux-mêmes et/ou d'expatriés de grandes firmes implantées dans les pays du Sud, est susceptible de rebattre les cartes quant à la nature, au processus et aux conséquences de l'expatriation (Collings et al., 2007; Cerdin et Le Pargneux, 2010; Zikic et al., 2010). L'émergence de ces expatriés «non-traditionnels » devrait conduire à de nouvelles approches prenant en compte les nouveaux profils mais aussi l'évolution des populations «traditionnelles » d'expatriés ainsi que les relations s'établissant entre ces catégories de plus en plus hétérogènes.

Cet article contribue à montrer que la considération conjointe d'expatriés issus des pays développés et de pays en développement rend possible une comparaison et une plus grande compréhension des trajectoires personnelles et professionnelles, de la mobilité sociale (Sorokin, 1927) de ces groupes. La communauté d'expatriés, comme tout groupe social, est organisée selon une hiérarchie, divisée en groupes et sous-groupes et caractérisée par des relations de pouvoir (Portes et al., 1999). L'étude de cette hiérarchie et de ces relations de pouvoir permet de comparer les situations de différents «types » et «classes » selon leur profil et les formes de capital dont ils disposent.

Quels sont les effets de l'expatriation, en particulier dans un pays à risque tel que le Nigeria, sur la position sociale et le statut de managers de pays «développés» et «en développement»?

\section{Mobilité géographique, socio-professionnelle et capitaux}

L'objectif est ici de montrer que la mobilité géographique (i.e., l'expatriation), conduit à des mobilités socio-professionnelles ascendantes (c'est-à-dire à une promotion intra-organisationnelle et/ou l'évolution d'un niveau socio-économique ou d'une classe sociale à une autre) d'intensité et de durabilité variées, en grande partie selon l'origine nationale et socioéconomique des expatriés. On observe ainsi l'existence d'un «plafond de verre de l'expatriation » (Insch et al., 2008) limitant la mobilité ascendante d'expatriés de pays en

1. Le vocable expatrié est largement usité dans le monde de l'entreprise. Un expatrié est étymologiquement une personne «qui a quitté sa patrie», avec une ambiguïté première sur le lien le rattachant à cette patrie : s'agitil du pays dont il a la nationalité ou de celui dont il est originaire? Une deuxième ambiguïté réside dans le terme «quitter», qui a une connotation définitive, ou de très long terme, la durée nécessaire pour pouvoir parler d' «expatriation» n'étant néanmoins pas définie. Cette durée varie en fait selon l'institution ou l'organisation qui définit l'expatrié. D'un point de vue juridique une personne n'est expatriée que si elle est enregistrée comme résidente dans un pays autre que le pays dont elle est ressortissante, c'est-à-dire dispose d'un visa et d'une carte de résident dans ce pays (valable deux ans pour le Nigeria). C'est loin d'être le cas de tous les étrangers travaillant au Nigeria dont beaucoup restent résidents de leur pays d'origine/nationalité et ne disposent au Nigeria que d'un visa business ou d'un permis de travail temporaire.

2. La condition d' «expatrié» représente un statut administratif visà-vis de l'entreprise, défini par le droit français. L'expression désigne alors une personne ayant un contrat de travail d'expatrié, situation très développement et de certains expatriés de pays développés au sein de multinationales. Cette recherche montre la reproduction à un niveau international de relations de pouvoir entre dominants et dominés par le biais des hiérarchies du commerce international (Gherardi et Pierre, 2010).

Les changements en termes de position sociale et de statut associés à des variations de différentes formes de capital (financier, social, culturel et symbolique) sont analysés parmi une population expatriée avec l'hypothèse que le pays d'origine a une influence majeure sur les différentes formes de capital accumulées au cours de l'expatriation. Cette origine détermine les formes de capital accumulé avant l'expatriation ainsi que le type de contrat que les expatriés obtiennent dans l'entreprise. Ces deux éléments influencent à leur tour la capacité des expatriés à accumuler du capital au cours de l'expatriation ainsi qu'à élever leur statut 1) au sein du pays d'expatriation, 2) dans leur pays d'origine et 3) dans une communauté globale émergente d'expatriés ou une élite globale.

La définition des «expatriés » ${ }^{1}$ est ici volontairement large. Il s'agit de ressortissants étrangers travaillant pendant une période prolongée ( 3 mois au moins, durée minimum des contrats au Nigeria dans l'organisation étudiée ainsi que pour la plupart des multinationales) pour la filiale internationale d'une multinationale ou pour un projet spécifique développé par la filiale dans cette zone géographique. Le statut d'«expatrié » est légalement défini par un contrat d'expatriation $^{2}$ entre l'individu et l'entreprise. Cependant, la définition adoptée dans cette recherche englobe volontairement des individus ne bénéficiant pas de contrat d'expatriation et ne se borne donc pas à l'analyse d'expatriés «traditionnels ». Cette définition correspond à celle observée sur le terrain et utilisée par l'entreprise et sa filiale dans la base ressources humaines $(\mathrm{RH})$ ainsi que par les individus définissant leur statut et celui des autres. Cette définition nous permet de considérer tant des expatriés «traditionnels», c'est-à-dire des managers qualifiés issus de pays développés, envoyés dans un pays en développement, puis rapatriés ou envoyé dans un autre pays, que des expatriés «non-traditionnels» : individus du «Nord» ou du «Sud» mutés par des entreprises sous des contrats moins avantageux que celui d'expatrié ou expatriés de leur propre initiative.

spécifique ne concernant que quelques cadres. Le contrat d'expatriation est l'une des formules pour une entreprise française souhaitant envoyer un salarié dans une filiale étrangère. Ces formules sont :

- Pour les missions courtes (moins de deux ans) :

- Le détachement : maintien du contrat de travail avec la structure d'origine, mais celui-ci fait l'objet d'un avenant concernant la mission à l'étranger;

- Le séjour à l'étranger avec retours réguliers en France : aucune modification du contrat de travail.

- Pour les missions de plus de deux ans :

- L'expatriation : le contrat de travail avec la structure française est suspendu mais pas rompu, et un contrat est passé avec la filiale d'accueil;

- L'embauche en contrat local : le contrat de travail avec la structure française est rompu et un CDI est signé avec la filiale d'accueil. 
A un niveau individuel, les postes expatriés et les conséquences de l'expatriation ont souvent été considérés comme favorisant diverses formes de capital en interaction : capital humain ou culturel, social, de carrière et, plus récemment, symbolique (ex. Al Ariss et Syed, 2010). En termes de capital humain ou culturel, c'est-à-dire l'ensemble des capacités productives qu'un individu acquiert par accumulation de connaissances générales ou spécifiques, de savoir-faire, etc. (Becker, 1964) ou l'ensemble des ressources culturelles détenues par un individu (Bourdieu, 1979), les recherches existantes considèrent que l'expatriation fournit aux individus une foule d'expériences professionnelles et personnelles favorisant le développement de savoirs et compétences. L'approche par le capital social, compris comme «l'ensemble des ressources actuelles ou potentielles [...] qui sont liées à un réseau durable de relations plus ou moins institutionnalisées d'interconnaissance et d'interreconnaissance» (Bourdieu, $1980: 2$ ), a porté son attention sur les ressources, atouts disponibles pour les expatriés encastrés dans des réseaux relationnels et examiné la façon dont ces relations et ressources associées pouvait bénéficier aux expatriés, rapatriés et/ou à leurs entreprises. Une approche fréquente en sciences de gestion a été, par ailleurs, de considérer l'expatriation en termes de «capital de carrière», agrégeant les diverses formes précédentes, ou de gestion de carrière. Plusieurs études ont ainsi montré que les différentes formes de capitaux et notamment le capital de carrière dépendaient de processus de gestion des filiales et du siège et notamment de leur capacité d'absorption, capacité à gérer les expatriations et rapatriements et à tirer profit des expériences internationales des individus concernés (Bjorkman et al., 2004; Chang et al., 2012). Dans une telle perspective, l'expatriation est considérée en termes d'impact sur la carrière, comme un élément fondateur ou accélérateur de carrière ou, au contraire, comme un frein à l'évolution professionnelle des individus (ex. Al Ariss, 2010). Certains chercheurs (ex. Doherty and Dickmann, 2009) ont proposé de réintroduire la notion de capital symbolique (Bourdieu, 1972, 1980), toute forme de capital considéré comme de valeur par les autres acteurs d'un champ lui permettant d'exercer une domination légitime. Le capital de carrière et les capitaux économique, social et culturel peuvent ainsi être convertis en capital symbolique dès lors qu'ils ont une valeur aux yeux d'autrui. Selon Bourdieu (1972), tout groupe socio-économique est un champ de bataille où les acteurs/agents sociaux luttent pour imposer leur vision, défendre les formes de capital légitimes et ainsi maintenir (dans le cas des dominants) ou tenter d'améliorer leur position dans le champ (dans le cas des dominés).

Cette étude analyse en ces termes les conséquences de l'expatriation au Nigeria pour différentes populations :

- Les Expatriés originaires d'économies développées, d'Amérique du Nord ou d'Europe de l'Ouest par exemple :

- Expatriés de pays développés appartenant aux classes supérieures (groupe 1)

- Expatriés de pays développés appartenant aux classes moyennes (groupe 2)
- Les Expatriés du «Sud», i.e. d'économies à revenus moyens ou faibles tels que les pays d'Afrique, du MoyenOrient, de certains pays d'Europe de l'Est et d'Amérique Latine :

- Expatriés du «Sud» appartenant aux classes moyennes (groupe 3)

- Expatriés du «Sud» appartenant aux classes modestes (groupe 4)

Au sein de chacun de ces groupes définis en fonction de la quantité de capital de différentes formes identifiée, certains sont envoyés au Nigeria en contrat d'expatriation (Groupe 1 pour la plupart) et les autres, en majorité, sont envoyés par l'entreprise avec des contrats plus précaires et moins intéressants que les contrats d'expatriés, ou expatriés à leur propre initiative (entrepreneurs sous-traitant pour l'entreprise notamment).

\section{Contexte, Méthodologie et Données}

Cette étude se fonde sur une recherche terrain de 18 mois (huit mois au Nigeria, dix mois en France) réalisant une sociologie des relations d'affaires franco-nigérianes. Cinq mois consécutifs ont été dédiés par l'un des chercheurs à l'observation d'expatriés au sein d'une multinationale (ici appelée Peironet) ayant sa maison-mère en France, une filiale au Nigeria et un projet (ici appelé Bouscatel) administrativement et légalement indépendant de la filiale nigériane.

18 entretiens semi-directifs d'une durée de 60 à 120 minutes ont été conduits en France et au Nigeria, en anglais, français et espagnol. Ceux-ci ont été menés selon une grille thématique visant à recueillir des informations sur 1) Les trajectoires sociales et spatiales des enquêtés 2) Leur profil détaillé au moment de l'enquête 3) Les motivations ou événements indépendants de leur volonté les ayant amené au Nigeria 4) Les raisons pour lesquelles ils restent dans ce pays, ou au contraire souhaitent en partir, leurs projets pour l'avenir 5) Les difficultés rencontrées et les appuis dont ils disposent dans la réalisation quotidienne de leur travail 6) Leur vie extra-professionnelle 7) Les relations entretenues avec un ensemble d'autres acteurs : leurs collègues, expatriés (de même nationalité / de nationalité différents) et nigérians, les expatriés d'autres entreprises, les nigérians d'autres entreprises (clients avec lesquels ils travaillent / non clients), etc. Ces informations ont permis d'établir divers types de trajectoires en fonction des nationalités des enquêtés, ainsi que différentes manières de prendre place au sein des entreprises pour lesquelles ils travaillent, de la communauté d'affaires franco-nigériane et de celle des expatriés au Nigeria, également fortement influencées par le pays d'origine (déterminant lui-même une certaine situation socio-économique).

L'observation des activités de la filiale et du projet a eu lieu sur une base quotidienne, en tant qu'auditeur qualité. Les missions confiées par l'entreprise étaient les suivantes : audit des relations avec le principal client nigérian, création d'un système de gestion de la documentation liée au principal 
projet commercial mené au Nigeria depuis sa création (contrats et correspondance principalement), compte-rendu sur les activités internes à la filiale (rythme des réunions et flux de communication interne principalement), préparation des documents nécessaires à un audit interne. Dans le cadre de la mission de mise au point d'une base de données permettant de classer et d'indexer la documentation écrite relative au principal projet commercial, l'accès à la correspondance a été donné par la direction de la filiale. Les lettres et e-mails échangés depuis le début du projet entre la filiale nigériane et le siège, et entre l'entreprise et son principal client au Nigeria ont été enregistrés dans une base de données (857 entrées). Bien que non formellement codés, l'analyse de leur contenu a fourni des informations sur la manière dont différents acteurs tenaient leur rôle professionnel en contexte de correspondance formelle, le ton employé en fonction des interlocuteurs concernés, etc.

Une analyse qualitative (codage) et quantitative (comptage) des fichiers RH, eux aussi fournis par la direction de la filiale dans le cadre d'une tâche consistant à répertorier les différents prestataires de service, a également été effectuée. A travers ces données, les chercheurs ont pu analyser les pays d'origine, statuts et positions, salaires, déplacements (professionnels et personnels), employeurs et contrats de travail de tous les expatriés au Nigeria et des employés nigérians de l'entreprise. Cela a permis d'obtenir des données quantitatives sur certaines composantes du profil des expatriés à l'étude.

En échange de la réalisation de ces missions confiées par l'entreprise, un accès était permis aux personnes, situations (réunions) et sources d'informations utiles pour la recherche en cours. Une grille d'observation de la vie quotidienne de l'entreprise a été utilisée, visant à saisir 1) la hiérarchisation s'opérant entre acteurs de la filiale; 2) le type de relations entretenus entre acteurs de la filiale et salariés de l'entreprise cliente; 3 ) les principes fédérant certains acteurs entre eux, et lesquels (nationalité, appartenance à une même entreprise, situation d'expatriation), et les principes faisant clivage et générant potentiellement des conflits. Cette observation a permis de compléter l'analyse des rôles au cours d'un ensemble de situations et d'obtenir une image très riche des relations existant en interne à la filiale mais aussi entre les expatriés et des acteurs extérieurs.

Les données recueillies par les différentes méthodes ont été intégrées par le biais de grilles thématiques au sein desquelles elles ont été fusionnées. Les principaux thèmes pertinents pour cet article ont concerné la gestion du personnel (nigérian et expatrié), les hiérarchisations formelles et informelles, les trajectoires des individus.

Ces méthodes de travail ont été adaptées en s'inspirant de trois grands domaines de recherche : les enquêtes en entreprises, les enquête auprès des «élites sociales », caractérisée par une importante distance symbolique et matérielle entre le chercheur et ses enquêtés, et les enquêtes en terrain à risque, impliquant de déployer le dispositif méthodologique choisi dans un cadre garantissant la sécurité physique du chercheur. Les enquêtes en entreprises sont facilitées par les techniques d'observation participante qui permettent au chercheur d'y avoir également une position de salarié interne (Flamant, 2005), d'où le choix de cette technique. Les enquêtes auprès d'élites sociales et/ou politiques, autre courant ayant influencé les choix méthodologiques de cette recherche, impliquent des difficultés d'accès et de communication avec les enquêtés qui demandent un soin particulier dans la prise de contact, la présentation de la recherche, et la manière de guider l'entretien pour recueillir la somme d'informations souhaitée tout en n'adoptant pas une posture trop directive (Laurens, 2007). Enfin, les enquêtes en terrain où existe un risque physique présentent des contraintes spécifiques en ce qu'elles sont marquées par un impératif de sécurité limitant certains déplacements, l'accès à certaines zones, etc. Les travaux à ce sujet mettent à jour que le contexte de risque physique fort a une influence importante sur les opportunités de déploiement du dispositif méthodologique et la construction de l'objet (Girard et Lagumier, 2006), éléments qui ont également marqué cette recherche.

Grâce à cet ensemble de méthodes, des indicateurs visant à évaluer les niveaux de capitaux des différents individus avant et pendant expatriation ont pu être mis en place. Pour le capital économique, il s'agit du niveau de salaire et éventuellement de revenus annexes connus; pour le capital culturel il s'agit du niveau de diplôme; pour le capital social, l'indicateur retenu est la possibilité avérée pour l'individu de changer de position (de poste, d'entreprise, de pays, de statut) grâce aux liens sociaux qu'il cultive (voire notamment la colonne «cooptation» du Tableau 2). Le capital symbolique enfin, est notamment mesuré à partir de l'appartenance à des cercles, clubs..., au nombre de fois et au type de circonstances (séminaires, réceptions, cérémonies officielles...) où l'individu est cité par ses pairs ou des personnes d'autres milieux (hommes politiques par exemple), analysé comme un signe de légitimité voire de prestige.

\section{Résultats - Impact de l'expatriation et de la nationalité sur le statut et le capital}

\section{NATIONALITÉ, STATUT ET CAPITAL AVANT EXPATRIATION}

L'origine nationale fonde des inégalités en termes de capital avant l'arrivée des expatriés dans l'entreprise au Nigeria. Leur choix de travailler au Nigeria est le résultat de contraintes liées à ces inégalités de capital. Les expatriés des pays émergents sont pour la plupart confrontés à des difficultés financières les poussant à quitter leur pays d'origine ou de résidence. Les expatriés des pays plus développés ont quant à eux des difficultés à s'élever professionnellement dans leur pays d'origine mais ne manquent pas de capital économique. Les profils que nous avons définis et leurs principales caractéristiques sont synthétisés dans le Tableau 1.

Le schéma et les tableaux suivants récapitulent par ailleurs l'origine géographique de l'employeur (Schéma 1), les nationalités et modes de recrutement des expatriés présents au moment de l'enquête (Tableau 2) et le type de contrats des principaux groupes d'expatriés (Tableau 3 ) 


\section{TABLEAU 1}

Appréciation des niveaux de capitaux avant expatriation, lien avec Peironet avant expatriation et cause principale d'expatriation selon les profils d'expatriés

\begin{tabular}{|c|c|c|c|c|c|c|}
\hline Profil & $\begin{array}{c}\text { Capital } \\
\text { économique }\end{array}$ & $\begin{array}{c}\text { Capital } \\
\text { culturel } \\
\text { (diplôme) }\end{array}$ & $\begin{array}{l}\text { Capital } \\
\text { social }\end{array}$ & $\begin{array}{c}\text { Capital } \\
\text { symbolique }\end{array}$ & Lien avec Peironet & Décision d'expatriation \\
\hline Groupe 1 & ++ & ++ & ++ & ++ & $\begin{array}{c}\text { Cadres dirigeants } \\
\text { au siège }\end{array}$ & $\begin{array}{l}\text { Recherche d'évolution } \\
\text { professionnelle / } \\
\text { problèmes personnels }\end{array}$ \\
\hline Groupe 2 & + & + & + & + & $\begin{array}{l}\text { Cadres } \\
\text { intermédiaires, } \\
\text { ingénieurs, sous- } \\
\text { traitants dans les } \\
\text { filiales }\end{array}$ & $\begin{array}{l}\text { A la recherche d'évolution } \\
\text { professionnelle + } \\
\text { recherche d'opportunité } \\
\text { financière / problèmes } \\
\text { personnels }\end{array}$ \\
\hline Groupe 3 & - & + & + & - & $\begin{array}{l}\text { Cadres } \\
\text { intermédiaires, } \\
\text { ingénieurs, sous- } \\
\text { traitants dans les } \\
\text { filiales }\end{array}$ & $\begin{array}{c}\text { Nécessité économique + } \\
\text { recherche d'opportunités } \\
\text { financières et } \\
\text { professionnelles }\end{array}$ \\
\hline Groupe 4 & - & - & - & - & Sans lien & Nécessité économique \\
\hline
\end{tabular}

\section{SCHÉMA 1}

\section{Origine géographique de l'employeur et type de contrat des principaux groupes d'expatriés de Peironet}

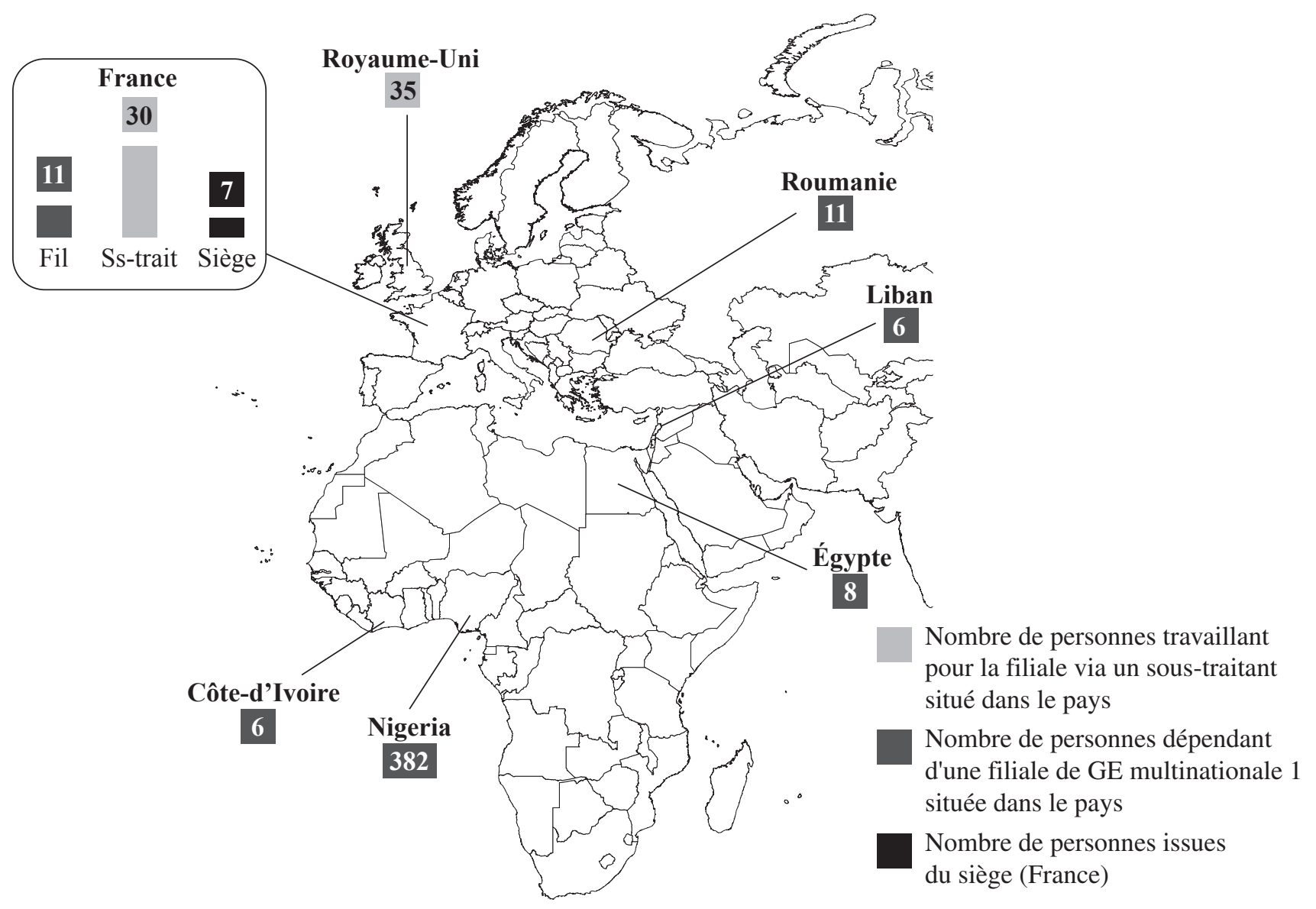


TABLEAU 2

Nationalité et mode de recrutement des principaux groupes d'expatriés à Peironet

\begin{tabular}{|c|c|c|c|c|c|c|}
\hline $\begin{array}{l}\text { Pays dont les } \\
\text { employés sont } \\
\text { nationaux }\end{array}$ & Nombre & $\begin{array}{l}\text { Nationalité } \\
\text { précédente }\end{array}$ & Nombre & $\begin{array}{l}\text { Recrutés } \\
\text { au siège }\end{array}$ & Cooptés & $\begin{array}{l}\text { Recrutés «classiquement» } \\
\text { (dans une filiale, via un sous- } \\
\text { traitant, ou par annonce) } \\
\text { - Information indiquée } \\
\text { lorsqu'elle est disponible }\end{array}$ \\
\hline \multicolumn{7}{|l|}{ Simple nationalité } \\
\hline Algérie & 1 & & & & & \\
\hline Etats-Unis & 1 & & & & & \\
\hline Brésil & 1 & & & & & \\
\hline Cameroun & 2 & & & & & 1 \\
\hline Costa-Rica & 1 & & 2 & & 1 & 1 \\
\hline Equateur & 2 & & & & & \\
\hline Egypte & 8 & & & & & 8 \\
\hline \multirow{5}{*}{ France } & \multirow{5}{*}{18} & & & 14 & 1 & 3 \\
\hline & & Sénégal & 1 & 1 & & \\
\hline & & $\begin{array}{l}\text { Côte- } \\
\text { d'Ivoire }\end{array}$ & 1 & 1 & & \\
\hline & & $\begin{array}{l}\text { Afrique } \\
\text { noire }\end{array}$ & 1 & 1 & & \\
\hline & & Liban & 1 & & & 1 \\
\hline Inde & 4 & & & & & \\
\hline Italie & 2 & & & 1 & & 1 \\
\hline Liban & 6 & & & & & \\
\hline Roumanie & 11 & & & & & 11 \\
\hline \multicolumn{7}{|l|}{$\begin{array}{l}\text { Double nationalités } \\
\text { et cas particuliers }\end{array}$} \\
\hline Argentine/Italie & 2 & & & & 2 & \\
\hline GB/Nigeria & 1 & & & & & 1 \\
\hline $\begin{array}{l}\text { Réfugié politique en } \\
\text { France }\end{array}$ & 2 & Cuba & 2 & & 1 & 1 \\
\hline
\end{tabular}




\section{TABLEAU 3}

\section{Type de contrat et profils d'expatriés}

\begin{tabular}{|c|c|}
\hline $\begin{array}{l}\text { Type de } \\
\text { contrat }\end{array}$ & Profil \\
\hline Expatrié & $\begin{array}{c}\text { Expatriés de «Pays Développé », «du } \\
\text { Nord» de «Classe Supérieure » (Groupe 1) }\end{array}$ \\
\hline $\begin{array}{l}\text { Transfert } \\
\text { d'une autre } \\
\text { filiale }\end{array}$ & $\begin{array}{c}\text { Expatriés de «Pays Développé», «du } \\
\text { Nord», et de «Pays en Développement», } \\
\text { du «Sud» de «Classe Moyenne» } \\
\text { (Groupe } 2 \text { et Groupe } 3 \text { ) }\end{array}$ \\
\hline $\begin{array}{l}\text { Sous- } \\
\text { traitant }\end{array}$ & $\begin{array}{c}\text { Expatriés de «Pays Développé», «du } \\
\text { Nord», et de «Pays en Développement», } \\
\text { «du Sud» de «Classe Moyenne» } \\
\text { (Groupe } 2 \text { et } 3 \text { ) et Expatriés de «Pays en } \\
\text { Développement», «du Sud } » \text { de «Classe } \\
\text { Inférieure } »(\text { Groupe } 4)\end{array}$ \\
\hline
\end{tabular}

Le type de contrat obtenu, conditionné en partie par l'origine géographique, est également révélateur de la place différente des individus dans la hiérarchie de l'entreprise et, plus largement, par les avantages et le statut qui lui sont rattachés, dans la communauté expatriée. C'est une première étape et un premier facteur dans la hiérarchisation qui s'opère entre les individus.

\section{NATIONALITÉ, STATUT ET CAPITAL LORS DE L'EXPATRIATION}

Groupe 1 (expatriés des pays développés appartenant aux classes supérieures)

\section{Capital économique}

Les membres de ce groupe bénéficient tous de contrats d'expatriation avantageux auprès du siège de Peironet ou d'une filiale étrangère. Leur contrat leur garantit de retrouver leur poste ou un poste équivalent à leur retour. On dénombre dix expatriés de cette catégorie dans l'équipe-projet Bouscatel, dont le Directeur Opérationnel de la filiale. Tous touchent entre $€ 7000$ et $€ 10000$ mensuels, en supplément de leur salaire avant expatriation (ce qui représente une augmentation de 70 à 100 pourcent). Ils bénéficient également d'avantages en nature (logement, transport, etc.), ce qui leur permet d'économiser une part importante de leurs revenus, d'investir dans leur pays d'origine ou de résidence (souvent la France) :

3. «Certaines nationalités se sentent supérieures, peut-être à cause de la manière dont elles sont traitées. Ça peut créer des conflits avec des nationalités qui ne sont pas aussi bien traitées. Au début les choses allaient bien. Mais ensuite d'autres personnes sont arrivées et elles ont obtenu des privilèges, ces privilège étaient une mauvaise chose. Les achat de propriétés, voitures, biens de luxe, etc. et de jouir d'une vie très confortable au Nigeria.

\section{Capital social}

Le fait de travailler dans ce cadre permet à ces expatriés d'accroître leur réseau professionnel. Leurs positions élevées dans la hiérarchie organisationnelle leur donnent l'opportunité de créer et de développer des relations au sein de l'organisation (cadres dirigeants, PDG). Leurs fonctions de représentation de l'entreprise au Nigeria sont pour leur part un support pour des relations avec l'extérieur : responsables politiques nigérians (membres de la Chambre de Commerce FrancoNigériane par exemple), autres cadres étrangers et diplomates. L'accumulation de capital financier facilite également le développement d'un tel réseau, permettant notamment de multiplier les invitations à dîner, d'organiser des soirées et de participer à certaines activités culturelles et sportives onéreuses réservées à une élite au capital culturel «légitime » (navigation de plaisance, pêche au gros, golf, etc.).

\section{Capital symbolique}

Cette catégorie d'expatriés gagne du prestige en travaillant dans un lieu réputé dangereux et également parce que le projet auquel ils participent est financièrement l'un des cinq plus importants du groupe dans le monde. Ils sont également considérés comme légitimes par leurs subordonnés nigérians; leurs hautes positions dans la structure locale leur donnent droit à un certain respect propre à «ceux qui font fonctionner les choses» («those making things work»). Des interviewés notent néanmoins que qu'il n'en a pas toujours été ainsi et déplorent l'arrogance de certains :

Some nationalities feel superior, maybe because of the way they are treated. It can create conflicts with others who are not treated so well. At the beginning, things were ok. But then, other people came, and gained privileges. These privileges were bad. Things began to get worse and worse, and there were lots of conflicts. [...] Now it is normal again. But it can escalate again, depending on the person who will have power ${ }^{3}$. (Ingénieur nigérian, 30 ans, homme, entretien formel)

La fréquence des contacts entretenus avec des Nigérians de classe supérieure (hauts dirigeants rencontrés lors d'activités professionnelles et extra-professionnelles) due à leur fonction de représentation constitue la principale différence entre ce groupe et les autres expatriés. Cela accroît leur légitimité auprès de l'ensemble des Nigérians :

[Notre client] ne fait confiance à personne. Il a identifié chez nous un certain nombre de personnes, qui sont le président, [Un responsable en France], et moi. Et il ne veut pas d'autre interlocuteur, hein! [...] Moi j'essaie de

choses ont commencé à aller de plus en plus mal, il y avait beaucoup de conflits [...]. Maintenant les choses sont revenues à la normale, mais ça peut empirer de nouveau, en fonction de la personne qui aura le pouvoir.» (Traduction libre des auteurs) 
lier des relations plus personnelles. [...] J'essaie de l'inviter à ma table, de le recevoir. Ça se passe très bien. (Chef de projet français, 50 ans, entretien formel).

De plus, ces expatriés organisent fréquemment de somptueux évènements et soirées aux adresses les plus luxueuses ce qui leur permet de montrer leur supériorité financière et leur pouvoir au sein de la communauté. Ainsi, les membres du Groupe 1 recréent au sein de la communauté expatriée la position dominante dont ils jouissent dans leur pays d'origine, et ce avec d'autant plus de facilité et un contraste d'autant plus évident que les classes moyennes sont potentiellement importantes numériquement mais encore en émergence et extrêmement hétérogènes au Nigeria.

Groupes 2 et 3 : Expatriés des classes moyennes des pays développés et $d u$ «Sud»

\section{Capital économique}

Ces catégories d'expatriés n'ont pas formellement de contrat d'expatriation. La plupart sont envoyés par une autre filiale (Egypte, Roumanie, Côte d'ivoire, agence de l'entreprise en France...) sans statut d'expatriés mais reçoivent une indemnité journalière de $€ 7000$ mensuels qui s'ajoute à leur salaire de base. D'autres sont employés par des sous-traitants et travaillent avec un statut de consultants. Dans ce cas, ils touchent en moyenne $€ 3600$ de plus que leur salaire de base grâce à une indemnité journalière de $€ 120$ avec quelques avantages en nature (transport). Pour ceux issus des pays développés, cette augmentation peut représenter jusqu'à 200 pourcent de leur revenu initial. Pour ceux venant des pays du Sud, elle représente entre 360 et 700 pourcent de plus.

Les expatriés des classes moyennes du «Sud» envoient la majorité de leurs revenus à leurs familles afin de satisfaire leurs besoins essentiels (logement, alimentation, éducation, etc.) alors que ceux issus des pays du «Nord», gagnant suffisamment avant expatriation pour assurer de tels besoins, emploient ce surplus d'argent pour s'offrir des conditions de vie confortables au Nigeria, économiser ou investir.

\section{Capital social}

Le fait de travailler et de vivre dans un nouvel environnement offre à ces expatriés des classes moyennes la possibilité de renforcer des réseaux déjà en partie établis, notamment par le biais de leurs études. Contrairement aux expatriés des classes supérieures, ils ne représentent pas l'entreprise auprès de parties prenantes extérieures et leurs relations avec les Nigérians sont de ce fait peu fréquentes et souvent conflictuelles. Les réseaux de ces «classes moyennes» sont généralement constitués d'autres expatriés rencontrés durant des évènements professionnels (expositions, salons, etc.) ou extra-professionnels (bars, restaurants). Les liens les plus forts sont souvent établis avec des compatriotes ou des

4. «Les expatriés sont toujours aux postes principaux. Par exemple à la comptabilité. Le problème ne vient pas des Nigérians : bien sûr qu'on comprend la comptabilité! Mais les entreprises ne veulent pas individus parlant la même langue maternelle. Ces liens leur procurent d'autres opportunités professionnelles au Nigeria ou ailleurs (certains expatriés des classes moyennes ont ainsi quitté Peironet pour rejoindre un client nigérian ou un concurrent).

\section{Capital symbolique}

La légitimité de ces catégories d'expatriés apparaît relativement ambiguë. La plupart occupent des postes intermédiaires dans la filiale. Ils sont de ce fait pris en tenaille entre leurs supérieurs (appartenant pour la plupart au groupe 1) et leurs subordonnés (groupe 4 et employés locaux). Leur «utilité» et leur présence au Nigeria sont fréquemment mises en question par les employés locaux qui considèrent souvent qu'euxmêmes pourraient aussi bien assurer les mêmes responsabilités si l'entreprise et ses dirigeants les y autorisaient :

Expatriates always hold the main positions. Accounting for instance. The problem is not with Nigerians : yes, we do understand accounting! But they don't want us to have access to financial information, they keep it secret ${ }^{4}$. (Salarié nigérian, Project Planner, 30 ans, homme, entretien formel).

La concurrence entre managers appartenant à des échelons relativement proches dans la hiérarchie crée un sentiment d'insécurité et des rivalités (Elias et Scotson, 1975), entre locaux et expatriés en particulier. Ces derniers se sentent ainsi obligés de justifier leur présence au Nigeria ainsi que leurs revenus et les indemnités reçues.

Le seul groupe au sein duquel ces personnes peuvent se sentir totalement légitimes est la communauté d'expatriés rencontrés hors de l'organisation, lors de loisirs par exemple. Cependant, cette communauté reste fortement localisée géographiquement et temporellement. Les contacts et la légitimité développés en son sein ne durent que le temps du séjour au Nigeria ce qui explique que beaucoup tentent de rester dans le pays en négociant âprement le renouvellement de leurs contrats avec Peironet ou en cherchant d'autres opportunités :

Il y a des gens qui sont restés au Nigeria, qui ne pourront plus rentrer en France. Au Nigeria ce sont des petits rois, ils ont des domestiques, des cuisiniers. En rentrant en France, ils n'ont plus rien. (Ingénieur franco-vietnamien, 30 ans, homme, entretien formel).

Groupe 4 : Expatriés du «Sud» appartenant aux classes modestes

\section{Capital économique}

Certains de ces expatriés voient leurs revenus multipliés par sept lors de leur expatriation. Tous travaillent via des soustraitants car leur niveau d'études ne leur a pas permis d'être

qu'ils aient accès à l'information financière, elles gardent le secret. » (Traduction libre des auteurs). 
engagés dans leur pays d'origine par Peironet ou ses filiales. Leurs contrats d'expatriation sont généralement établis pour trois mois. Ils dépensent une part importante de leurs revenus pour se loger et expédient le reste à leur famille pour assurer leurs besoins élémentaires. Leur principal défi est de transformer une augmentation temporaire de leurs revenus en une stratégie financière à long-terme : ceux-ci enchaînent ainsi fréquemment les expatriations dans des pays à risque (en Afrique par exemple) afin d'accumuler du capital économique.

\section{Capital social}

Leurs réseaux initiaux étant relativement limités dans leur pays d'origine (où ils n'ont ni réseaux d'études, n'ayant pas fait d'études supérieures, ni réseaux professionnels, ayant fonctionné jusque-là par la «débrouille», s'appuyant sur un ou deux contacts clés), ceux-ci se développent considérablement lors de l'expatriation.

Lors de leur séjour au Nigeria, les membres de ce groupe développent leur réseau initial en faisant des rencontres au travail ou parmi la communauté d'expatriés. Ils développent des liens avec d'autres professionnels travaillant pour Peironet ou pour d'autres entreprises.

\section{Capital symbolique}

Comme les expatriés des classes moyennes, les membres du groupe 4 occupent des positions intermédiaires mais se sentent plus en position d'infériorité que ces derniers :

Nosotros somos el bajo nivel del management. Somos el front office, los que estamos en la mierda aquí en Nigeria. Cuando el cliente no está contento golpea nuestra cara. Después tienes un nivel intermediario que es [le chef de projet au Nigeria]. El alto nivel está en Francia ${ }^{5}$. (Project Manager argentin, 30 ans, homme, conversation informelle).

Comme les expatriés des groupes 2 et 3 , ceux des classes inférieures des pays en développement ressentent souvent le besoin de justifier leur présence au Nigeria, leurs postes et leurs conditions de travail bien supérieures à celles de leurs collègues nigérians. Toutefois, contrairement aux autres expatriés, étant donné le peu de légitimité dans leur pays d'origine, la situation à laquelle ils se trouvent confrontés au Nigeria représente plutôt une évolution positive pour eux. Leur manque de diplômes en particulier (condition sine qua non pour travailler dans une multinationale dans leur pays d'origine) n'apparaît pas aussi stigmatisant au Nigeria.

Leur capital économique leur permet de jouir d'un mode de vie hors d'atteinte dans leur pays d'origine. Ils partagent les loisirs des autres expatriés et sont riches vis-à-vis de la population locale qui les considère réciproquement comme «des expatriés comme les autres ».

5. «Nous sommes le bas niveau du management. On est le Front Office, ceux qui avons les pieds dans la merde ici au Nigeria. Quand le client n'est pas content c'est sur notre figure qu'il tape. Après tu as un

\section{SYNTHÈSE}

En ce qui concerne les expatriés issus de pays développés et de «classe supérieure», l'expatriation dans un pays à risque renforce le capital initial sous ses différentes formes. L'expatriation confirme et étend des positions socio-professionnelles déjà privilégiées dans deux contextes : le pays d'origine et le pays d'accueil. En ce qui concerne les autres expatriés, de tels postes favorisent l'accumulation rapide de diverses formes de capital et permettent un accès temporaire à un statut privilégié dans le pays d'accueil (Tableaux 4 et 5).

Cette accumulation ne permet toutefois pas de briser un «plafond de verre de l'expatriation » qui empêche la plupart des expatriés d'atteindre les positions les plus prestigieuses au sein de l'organisation comme au sein de la communauté d'expatriés (Schéma 2).

L'encadré suivant et le Tableau 6 synthétisent pour mémoire les principales conclusions.

\section{Discussion et conclusion}

L'étude de l'expatriation en pays à risque permet de dévoiler certaines relations de classe transnationales. Les arguments principaux développés ici sont 1) que les dynamiques relationnelles observées entre expatriés dans des pays à risque reflètent celles plus larges dans le cadre de la mondialisation et 2) réciproquement, que les trajectoires d'expatriation et les parcours personnels et professionnels à plus long terme des expatriés se trouvent fortement influencés par la reproduction de relations domestiques de classes qui se prolongent désormais à une échelle globale.

Les entreprises multinationales représentent une des principales forces internationales reconfigurant les relations sociales. A un niveau organisationnel, les structures des multinationales reflètent les inégalités structurelles entre groupes dominants et subordonnés (Embong, 2000). On observe un décalage important entre un discours vague, généralisant et positif au sujet de la «diversité» culturelle, nationale et ethnique, et l'organisation effective de cette diversité sur le terrain. Dans les discours d'entreprises (sites web, supports publics de communication, entretiens avec les cadres dirigeants), la diversité culturelle serait gage de dynamiques «positives», d'énergie, d'un travail plus efficace et d'une bonne ambiance. La question des différenciations, des hiérarchies et des rapports de pouvoir, voire des conflits basés sur les identifications nationales, culturelles et ethniques est occultée par ces discours qui décrivent des relations de type égalitaire entre les salariés originaires de différents pays. Cette étude invite, en portant sur la diversité dans le contexte d'une multinationale au Nigeria un regard empirique et non normatif, à réintroduire au sein de la diversité la

niveau intermédiaire qui est [le responsable du projet au Nigeria]. Le haut niveau il est en France.» (traduction libre des auteurs). 


\section{TABLEAU 4}

\section{Source et montant des revenus connus (capital économique)}

\begin{tabular}{|c|c|c|}
\hline Fonction/statut & Source de revenus & Montant des revenus \\
\hline Groupe 1 & $\begin{array}{l}\text { Salaire }+ \text { intéressement aux bénéfices de } \\
\text { l'entreprise ou du projet }+ \text { Daily allowance } \\
\text { (per diem) + avantages en nature : } \\
\text { appartement et voiture de fonction, non } \\
\text { imposition de certains revenus }\end{array}$ & $\begin{array}{l}\text { Salaire en fonction du grade et de l'ancienneté } \\
\text { (Entre } 2500 \text { et } 10000 \text { euros) } \\
+240 \text { dollars par jour per diem }\end{array}$ \\
\hline Groupe 2 & $\begin{array}{c}\text { Salaire + Daily allowance }(\text { per diem }) \\
+ \text { revenus du capital pour les chefs de petites } \\
\text { entreprises sous-traitantes }\end{array}$ & $\begin{array}{l}\text { Pour les salariés : Salaire en fonction de l'entreprise } \\
\text { qui embauche, entre } 1500 \text { et } 3000 \text { euros }+136 \\
\text { dollars par jour per diem } \\
\text { Pour les responsables de petites entreprises : } \\
1000 \text { euros environ + défraiement + une partie du } \\
\text { bénéfice, qui dépend du montant des contrats passés }\end{array}$ \\
\hline Groupe 3 & $\begin{array}{c}\text { Salaire + Daily allowance }(\text { per diem }) \\
+ \text { revenus du capital pour les chefs de petites } \\
\text { entreprises sous-traitantes }\end{array}$ & $\begin{array}{l}\text { Salaire en fonction de l'entreprise qui embauche, } \\
\text { entre } 500 \text { et } 1500 \text { euros, } \\
+136 \text { dollars par jour per diem } \\
\text { Pour les responsables de petites entreprises : } \\
1000 \text { euros environ + défraiement + une partie du } \\
\text { bénéfice, qui dépend du montant des contrats passés }\end{array}$ \\
\hline Groupe 4 & Salaire + Daily allowance (per diem) & $\begin{array}{c}\text { Salaire en fonction de l'entreprise qui embauche, } \\
\text { entre } 500 \text { et } 1500 \text { euros, } \\
+136 \text { dollars par jour per diem }\end{array}$ \\
\hline
\end{tabular}

\section{TABLEAU 5}

Evolution du Capital des Expatriés

\begin{tabular}{|c|c|c|c|c|c|c|c|c|}
\hline \multirow[t]{2}{*}{ Profil } & \multicolumn{2}{|c|}{ Capital économique } & \multicolumn{2}{|c|}{$\begin{array}{l}\text { Capital culturel } \\
\text { (diplôme) }\end{array}$} & \multicolumn{2}{|c|}{ Capital social } & \multicolumn{2}{|c|}{ Capital symbolique } \\
\hline & $\begin{array}{c}\text { Pays } \\
\text { d'origine }\end{array}$ & Nigeria & $\begin{array}{c}\text { Pays } \\
\text { d'origine }\end{array}$ & Nigeria & $\begin{array}{c}\text { Pays } \\
\text { d'origine }\end{array}$ & Nigeria & $\begin{array}{c}\text { Pays } \\
\text { d'origine }\end{array}$ & Nigeria \\
\hline Groupe 1 & ++ & +++ & ++ & ++ & ++ & +++ & ++ & +++ \\
\hline Groupe 2 & + & ++ & + & + & + & ++ & + & ++ \\
\hline Groupe 3 & - & ++ & + & + & + & ++ & - & ++ \\
\hline Groupe 4 & - & ++ & - & - & - & ++ & - & + \\
\hline
\end{tabular}


SCHÉMA 2

Poste, nationalité, âge et type de contrat des cadres de Peironet les plus haut placésau Nigeria - Réalisé à partir des données de terrain

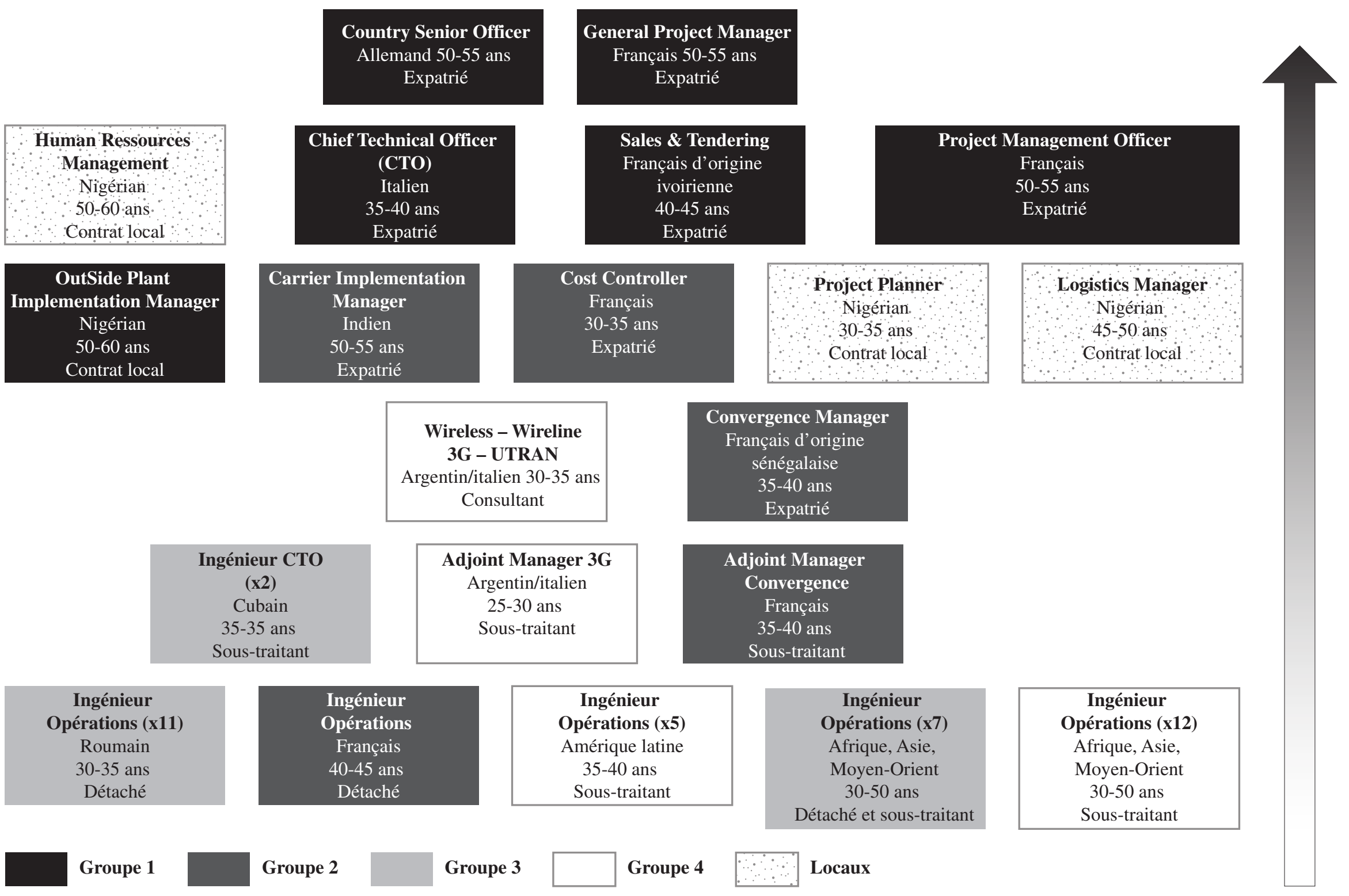




\section{ENCADRÉ 1}

\section{Synthèse de la démarche et des axes de conclusions}

Synthèse de la démarche et des principales conclusions

Population étudiée : individus issus des classes moyennes et supérieures des pays du «Nord» avec et sans contrat d'expatriation, et des classes moyennes et inférieures de pays du «Sud» sans contrat d'expatriation, travaillant au sein d'une même filiale d'entreprise située au Nigeria.

Méthodes : observation participante, entretiens, analyse des documents et fichiers RH, analyse de la correspondance interne et externe. Cadre méthodologique : enquête en entreprise, enquête auprès des élites, enquête sur des terrains à risques.

\section{Axes de conclusion :}

Sur la démarche d'expatriation : expatriation dans un pays à risques, systématiquement sous contraintes, avec des degrés de contraintes plus ou moins forts et des types de contraintes différents en fonction des origines géographiques et des profils sociaux des individus.

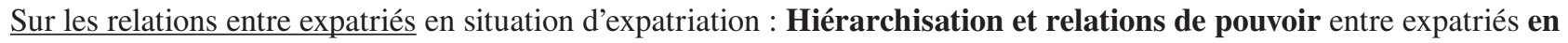
fonction de leur origine. Cette hiérarchisation passe notamment par l'obtention d'un type de contrat différent (expatrié, détaché, sous-traitant embauché par une autre entreprise ou indépendant), qui dépend partiellement de l'origine géographique. Elle se traduit en particulier par l'existence, au sein de l'entreprise d'un relatif «plafond de verre» de l'expatriation lisible dans la structure de l'organigramme (schéma 2), et hors de l'entreprise, par l'évolution au sein de cercles sociaux parfois différenciés et des contacts plus ou moins soutenus avec les hautes strates de la population locale et les milieux politiques.

Sur les effets individuels de l'expatriation : Mobilité sociale et professionnelle ascendante mais inégale en fonction des origines nationales et sociales des individus, ces profils étant caractérisés par une dotation initiale, puis une accumulation différenciées en termes de capitaux matériel, culturel, social et symbolique.

question des rapports de force dont elle est porteuse. Ces résultats font écho en particulier aux travaux sociologiques évoquant l'émergence d'une classe managériale ou capitaliste transnationale» (Sklair, 2001). La question sous-tendant ce travail était de savoir si une telle classe entrave la mobilité sociale et professionnelle de nouveaux venus, en particulier issus de pays en développement. Nos résultats montrent que l'intégration de ces nouveaux expatriés dans une classe dominante transnationale reste superficielle et temporaire. L'expatriation dans un pays à risque permet à tous l'accumulation rapide de capital économique, social et symbolique. Cependant, les parcours socio-professionnels observés illustrent que cette expatriation n'a pas la même portée symbolique selon les individus et que les formes de capital développées ne recouvrent pas la même validité, ne sont pas aussi transférables ni durables selon le pays d'origine et le statut social avant expatriation.

Le mythe de la mobilité vers des terres d'opportunités qui motive beaucoup à s'expatrier contraste avec la réalité marquée par au moins deux ensembles sociaux polarisés : l'un pour lequel l'expatriation n'est qu'une étape professionnelle plus ou moins obligée, un rite de passage pour accéder à de plus hautes fonctions et positions sociales; l'autre pour lequel l'expatriation est plutôt un choix personnel sous contraintes (faible niveau d'éducation ne permettant pas de décrocher un emploi rémunérateur; impossibilité de trouver du travail, ou un travail adapté à sa formation dans le pays d'origine, etc.), plus durable, permettant une mobilité sociale et professionnelle dans certaines limites et dans un périmètre géographique restreint.

La définition large des expatriés retenue dans ce travail, par opposition aux expatriés «juridiques », c'est-à-dire titulaires d'un contrat de travail d'expatrié, présente l'avantage non négligeable de permettre d'observer ce second ensemble, composé d'expatriés «non-traditionnels » généralement négligés par les recherches antérieures. Cette acception large n'est pas sans certaines limites : elle renvoie à une définition de l'expatrié considérée comme impropre d'un point de vue légal ou du point de vue de certaines organisations multinationales, elle rend difficile la distinction entre les catégories de mobilité que sont l'expatriation, l'immigration et le « voyage d'affaires» (où commence l'un, ou finit l'autre?). Cependant, pour traiter l'objet qui est le nôtre dans la perspective théorique choisie, et à condition d'opérer des distinctions précise au sein des «expatriés » en fonction de critères pertinents, cette définition a une portée heuristique et opérationnelle importante.

L'analyse de la mobilité et de l'expatriation notamment dans un pays à risques met au jour des contradictions. 


\section{TABLEAU 6}

\section{Synthèse des principaux résultats par catégorie de population}

\begin{tabular}{|c|c|c|c|c|}
\hline & $\begin{array}{l}\text { Origine sociale } \\
\text { et géographique }\end{array}$ & $\begin{array}{c}\text { Type de contrainte motivant } \\
\text { l'expatriation }\end{array}$ & $\begin{array}{l}\text { Type de } \\
\text { contrat } \\
\text { obtenu }\end{array}$ & Evolution des capitaux \\
\hline Groupe 1 & $\begin{array}{l}\text { Pays du } \\
\text { «Nord», } \\
\text { hautes classes } \\
\text { sociales }\end{array}$ & $\begin{array}{c}\text { Manque d'opportunités } \\
\text { professionnelles au siège de } \\
\text { l'entreprise ou dans la branche } \\
\text { se situant dans le pays où ils } \\
\text { sont nationaux / demande de } \\
\text { l'entreprise }\end{array}$ & Expatrié & $\begin{array}{c}\text { Moyenne, durable (les revenus } \\
\text { financiers notamment, sont épargnés } \\
\text { au moins en partie) }\end{array}$ \\
\hline Groupe2 & $\begin{array}{c}\text { Pays du } \\
\text { «Nord } » \\
\text { moyennes } \\
\text { classes sociales }\end{array}$ & $\begin{array}{c}\text { Pour ceux travaillant } \\
\text { au sein de l'entreprise : } \\
\text { manque d'opportunités } \\
\text { professionnelles au sein } \\
\text { de l'entreprise dans le } \\
\text { pays où ils sont nationaux. } \\
\text { Pour les sous-traitants } \\
\text { salariés ou indépendants : } \\
\text { manque d'opportunités } \\
\text { professionnelles ou } \\
\text { commerciales sur les marchés } \\
\text { nationaux }\end{array}$ & $\begin{array}{l}\text { Détaché } \\
\text { ou sous- } \\
\text { traitant }\end{array}$ & $\begin{array}{c}\text { Forte, durable (les revenus financiers } \\
\text { notamment, sont épargnés au moins } \\
\text { en partie) }\end{array}$ \\
\hline Groupe 3 & $\begin{array}{c}\text { Pays du } \\
\ll \text { Sud } », \\
\text { moyennes } \\
\text { classes sociales }\end{array}$ & $\begin{array}{l}\text { Pas de possibilité d'être } \\
\text { recruté dans le domaine } \\
\text { où sont diplômés, ou à des } \\
\text { salaires trop bas pour subvenir } \\
\text { aux besoins de leur famille. }\end{array}$ & $\begin{array}{l}\text { Détaché } \\
\text { ou sous- } \\
\text { traitant }\end{array}$ & $\begin{array}{c}\text { Forte, temporaire (les revenus } \\
\text { financiers notamment, sont dépensés } \\
\text { et conditionnés par le fait de } \\
\text { s'expatrier) }\end{array}$ \\
\hline Groupe 4 & $\begin{array}{c}\text { Pays du } \\
\text { «Sud», basses } \\
\text { classes sociales }\end{array}$ & $\begin{array}{c}\text { Manque d'éducation limitant } \\
\text { l'employabilité. Revenus } \\
\text { insuffisants pour subvenir à } \\
\text { leurs besoins et à ceux de leur } \\
\text { famille. }\end{array}$ & $\begin{array}{l}\text { Sous- } \\
\text { traitant }\end{array}$ & $\begin{array}{c}\text { Très forte, temporaire (les revenus } \\
\text { financiers notamment, sont dépensés } \\
\text { et conditionnés par le fait de } \\
\text { s'expatrier) }\end{array}$ \\
\hline
\end{tabular}

L'expatriation est souvent fortement valorisée dans le discours des entreprises comme par une partie de la littérature qui y voit un «accélérateur de carrière», un effet catalytique sur l'accumulation de différentes formes de capital et l'ascension professionnelle et sociale résultantes, à plus forte raison dans des pays où le caractère extrême de l'expatriation (de par les dangers, notamment physiques, potentiellement encourus) est censé amplifier le phénomène. La recherche a aujourd'hui du mal à expliquer des résultats où expatriation et évolution de carrière / promotion sociale et professionnelle sont de moins en moins systématiquement corrélés.

Cette étude contribue à un éclairage sociologique de ces variations dans la force du lien, en les expliquant en particulier par l'origine géographique des individus. On observe d'abord que, quelle que soit l'origine, la mobilité en pays à risque est toujours subie, contrainte. Cette contrainte provient soit de l'entreprise d'origine qui fait de l'expatriation une condition, un point de passage nécessaire pour «éprouver» les jeunes recrues et autres individus avant que ceux-ci n'accèdent éventuellement à de plus hautes responsabilités (cas de la plupart des expatriés des pays développés), soit des conditions de vie dans le pays d'origine, les individus en question se sentant prêts à prendre des risques pour tenter d'améliorer leurs conditions matérielles et celles de leurs familles (cas de la plupart des expatriés des pays en développement observés). D’autre part, on observe qu'en dépit d'un discours fréquent vantant pour tous les bénéfices potentiels d'une expatriation (enrichissement financier, possibilité d'obtenir un poste plus prestigieux et plus de responsabilités, image positive de «baroudeur», d'«aventurier» et compétences associées), l'origine géographique et sociale des individus reste, pour les originaires de pays du «Sud» et les moins dotés en capital social (relations) et culturel (diplôme) quel que soit leur pays d'origine, un stigmate que l'accumulation 
de toutes formes de capital lors de l'expatriation n'efface que temporairement et partiellement. «A l'exemple des différences de prestige entre écoles de commerce ou d'ingénieurs, les filières nobles de l'internationalisation (dans les pays les plus industrialisés) se distinguent de celles qui le sont moins » (Pierre, 2003, p.39). Une expatriation motivée/contrainte par l'entreprise (cas typique des expatriés des pays les plus développés) se trouve plus valorisée et valorisante socialement et professionnellement (évolution de carrière) qu'une expatriation motivée/contrainte par des raisons matérielles (cas typique des expatriés des pays moins développés). La réalité concrètement observée dans cette étude se révèle loin de l'image positive, «vulgate (managériale) planétaire dont sont remarquablement absents (les termes de) capitalisme, classe, exploitation, domination, inégalité » (Bourdieu et Wacquant, 2000). L'origine sociale et géographique reste le fondement de classes incluant/séparant les expatriés comme les autres acteurs de la mondialisation (Wagner, 2007).

On observe ici, sur un terrain gestionnaire, que la position et le positionnement social «se joue(nt) en partie dans la dimension spatiale, c'est-à-dire dans la capacité inégale qu'ont les individus et les groupes à retirer des usages de l'espace un certain nombre de ressources matérielles et symboliques et à les transmettre» (Veschambre, 2006, p.10) et que, inversement, cette dimension spatiale est dépendante de l'origine, de la position sociale de l'individu. Ces observations peuvent être mises en relation avec la réflexion autour des notions de dimension spatiale des rapports sociaux (Sechet et al., 2006), de capital spatial, et d'interaction entre capital spatial et capital social ${ }^{6}$. Plusieurs travaux (Levy, 1994, 2008; Pierre, 2003; Gherardi et Pierre, 2010) ont évoqué l'importance croissante d'un «capital spatial» défini de façon générale comme «l'ensemble des ressources, accumulées par un acteur, lui permettant de tirer avantage, en fonction de sa stratégie, de l'usage de la dimension spatiale de la société » (Levy, 1994). Plus précisément, on distingue un «capital spatial de position », inhérent à l'appartenance à un lieu et un «capital spatial de situation », lié à une aire, à un espace que l'individu s'approprie (Loudiyi et al., 2004) «par toutes sortes de mobilités, sans abolir les distances, mais en les maîtrisant» (Levy, 1994, p.95). La présente étude montre une inégalité persistante en termes de capital spatial selon l'origine géographique et sociale des individus. Si les expatriés des pays en développement acquièrent, au moins le temps de l'expatriation, une forme de capital spatial de position, ils restent relativement dépourvus de capital spatial de situation. Si ces individus, tolérés car nécessaires au fonctionnement de l'entreprise dans le pays d'accueil, s'intègrent, le temps de l'expatriation, à l'espace et au champ occupés par une certaine élite inter- ou trans-nationale (managers internationaux, diplomates, etc.) (Robinson et Harris, 2000, Rofe, 2003) et en retirent un certain nombre d'avantages, de capitaux, ils en maîtrisent moins les codes. La mobilité, entendue comme l'«aisance à

6. Nous remercions un évaluateur anonyme pour cette suggestion en particulier ainsi que pour ses commentaires et références proposées qui se mouvoir, à agir, à envisager par la pensée une portion de l'espace» et qui constitue «la dimension spatiale de l'habitus» (Tizon, 1996) leur est moins accessible qu'à leurs pairs des classes dominantes. Leur moindre capital économique, leur moindre insertion au sein des entreprises mondialisées et leur nationalité rendant plus difficile que pour d'autres le fait de voyager en règle vers certaines destinations, sont autant d'éléments les exposant à une mobilité moins large et moins choisie que celle des strates dominantes.

On observe une certaine «matérialité de l'espace» (Veschambre, 2006) : mobilité, pratiques du territoire, déplacements, lieux de résidence, de travail, de loisir, etc. associés à divers capitaux tangibles, mais aussi à sa «composante idéelle» (représentations de l'entreprise, du monde dans lequel s'inscrit l'activité, stratégies d'expatriation, de mobilité professionnelle des individus, etc.) qui structurent l'espace comme «composante sociale», fondent le social comme «spatialement constitué» (Chivallon 2000). Se confrontent ainsi deux images ou imaginaires de l'international et de la mobilité (Vilaça, 2001) : l'une, plutôt pessimiste, associant mobilité et exil (réfugiés, sans papiers et autres «victimes » de la mondialisation) selon laquelle les rapports sociaux à l'international ne permettent in fine que d'assurer la reproduction sociale (Fournier, 2008), l'autre, plutôt optimiste, considérant que la mobilité spatiale peut influencer positivement la mobilité sociale en permettant aux individus mobiles d'acquérir une position (plus) élevée dans la hiérarchie sociale en intégrant un stock d'expériences ainsi que les capacités d'agir dans un ensemble en échange de différents capitaux (symboliques, culturels, économiques, sociaux...) (Levy, 2008). Ce travail s'inscrit plutôt dans la première perspective, celui d'un champ international ou global peuplé de classes déjà établies de longue date dans les pays d'origine, d'une hiérarchie sociale qui ne fait que trouver une extension globale (Wagner, 2007). Ce champ constitué de multiples acteurs aux profils et origines géographiques et sociales de plus en plus variés s'avère fort conservateur bien qu'émergent de plus en plus, non pas une communauté globale uniforme, mais de multiples communautés, notamment celle des expatriés des pays émergents, forme de «gentrifying class» temporaire (Rofe, 2003) fortement localisée qui se heurte aux classes dominantes (des pays les plus développés) lorsqu'ils tentent de se projeter au-delà du local.

Le choix fait d'approcher notre objet sous l'angle de l'influence et de l'évolution des capitaux en situation d'expatriation, croisé avec l'origine nationale des acteurs, s'avère riche pour analyser les trajectoires de différents types d'expatriés. Pour des raisons de clarté et de rigueur, nous nous en sommes tenus ici à l'analyse de ces critères. Cela ne doit pas occulter l'existence d'autres éléments constitutifs de l'identité pouvant influencer l'évolution des sujets lors de l'expatriation. Par exemple, la culture professionnelle constituerait une piste complémentaire permettant d'enrichir et de prolonger

ont constitué le point de départ de cette partie de la discussion. 
l'analyse. Les expatriés des groupes 1, 2 et 3 sont majoritairement ingénieurs ou cadres de formation qui continuent d'exercer leur métier au Nigeria, même si celui-ci peut y prendre des modalités très différentes de ce qu'ils ont connu dans leur pays d'origine. Le groupe 4 est plus hétéroclite et contient majoritairement des personnes non formées à la base aux métiers exercés au Nigeria. Cette fracture professionnelle est susceptible de venir renforcer, au début de l'expatriation, les frontières de classe et de nationalité. Au fur et à mesure que les acteurs du groupe 3 apprennent leurs métiers et se familiarisent avec les codes et le jargon, la culture professionnelle peut constituer un facteur unificateur venant contrecarrer les barrières de classe et de nationalité. Ces pistes mériteraient d'être explorées de manière systématique dans le contexte nigérian ou dans d'autres cas.

Insistons également sur le fait que la présente étude a mis au jour ce phénomène dans un cadre localisé dans le temps et l'espace. Il n'existe pas une dynamique univoque liée à la possession et à l'accroissement de capitaux en tous genres, mais bien des mécanismes complexes qui se combinent, ici des ascensions sociales différenciées pour les différentes strates de la population considérée, doublée d'un certain maintien de l'ordre existant au départ, avant mobilité. Le cas présenté ici peut aussi être mis en perspective par rapport à d'autres cas présents dans la littérature, ce qui offre plusieurs prolongements possibles à la recherche.

Les écrits sur le «plafond de verre» sont un premier corpus auquel confronter cette étude. L'existence de ce plafond a été prouvée par maints travaux portant sur le critère du sexe, de l'âge, de la «race» et de l'éducation, à l'échelle domestique ou dans le cadre de l'expatriation (voir McNulty et al., 2009 pour une revue). En revanche, peu de travaux ont étudié l'existence d'un tel plafond selon l'origine géographique et sociale et dans le cas d'expatriés, dans des entreprises françaises ou d'autres. Nos résultats viennent enrichir les recherches sur cette thématique et apporter un contrepoint à une littérature managériale encore limitée mais souvent «optimiste». Ce cas d'individus évoluant au sein d'une multinationale française au Nigeria reste cependant une «figure du possible». Une perspective de recherche future serait d'étudier d'autres individus aux mêmes origines évoluant dans des entreprises autres que françaises et/ou ayant d'autres politiques au sujet de l'expatriation et la gestion de carrière de ses personnels.

Par exemple, et c'est une seconde thématique à laquelle pourrait être confrontée ce travail, certaines entreprises mettent en place des politiques de multinationalisation de leur direction et de leur encadrement. Un certain nombre d'entreprises, en particulier les plus grandes, ont développé des politiques de «multinationalisation» de leurs dirigeants et managers consistant à promouvoir la mobilité internationale de ces personnes en les envoyant auprès de filiales ou de partenaires étrangers (ex. Peterson et al., 1993). Ces politiques se traduisent le plus souvent par la promesse d'un avancement de carrière, des conditions financières avantageuses et un soutien à la famille de l'individu, à sa socialisation dans le pays d'accueil. Des études récentes (McNulty et al., 2009; Doherty et Dickmann, 2012) portant sur le «retour sur investissement» de ces politiques, pour les entreprises comme pour les individus, concluent seulement que celui-ci s'avère « complexe», difficile à interpréter et fluctuant. Ces politiques de «multinationalisation» peuvent également porter sur la sélection et nomination de ressortissants étrangers issus de pays «en développement» à des postes de direction, dans le cadre notamment d'une promotion de la «diversité». Les travaux sur la question (Hartmann, 2011) montrent un faible enthousiasme pour ces politiques et un maintien manifeste d'élites traditionnelles, c'est-à-dire d'hommes nord-américains ou européens, de type caucasien, d'au moins une quarantaine d'années, formés dans certaines institutions (grandes écoles en France, Cambridge, Oxford, en Angleterre, universités de l'Ivy League aux Etats-Unis, etc.) au sein des conseils d'administration et des directions des grandes entreprises. Nos résultats contribuent à montrer que la prégnance de ce type d'élite à la tête des entreprises n'est pas généralisable à tous les contextes mais que la diversification des élites commence dans des «niches» particulièrement difficiles et n'empêche pas le maintien d'une forte hiérarchisation.

On peut également se demander, avec la part croissante des pays émergents et la remise en cause des hiérarchies nationales dans l'économie mondiale, combien de temps encore prendra l'arrivée à des postes de direction, l'ascension professionnelle et sociale d'individus issus de pays encore considérés il y a peu comme «sous-développés». Que ce soit par la mise en place de politiques d'entreprises ou par adaptation de ces dernières à un monde où de plus en plus de partenaires, concurrents, clients, fournisseurs, etc. sont issus de ces pays, les hiérarchies sociales et professionnelles sont vouées à évoluer.

D'autres prolongements pourraient être envisagés. L'un consisterait à étudier de manière systématique la question du recrutement des expatriés, par exemple à partir de banques de données d'annonces en ligne, afin de déterminer les postes ouverts, leur localisation, leurs caractéristiques. Il s'agirait ensuite de tracer les filières de recrutement sur ces postes, le profil des personnes recrutées et leur devenir une fois dans le poste. Cela permettrait, à plus large échelle, de confirmer ou de nuancer notre thèse selon laquelle, en fonction du pays d'origine, les personnes n'ont pas toutes accès aux mêmes opportunités de postes, et que les conséquences de l'expatriation sont dès lors différenciées. La durée d'expatriation et ses impacts sur l'accumulation de capitaux pourraient être un autre angle à approfondir. La durée peut être un facteur déterminant dans la possibilité d'accumuler du capital. Dans cette étude, une expatriation qui dure permet une certaine ascension sociale et une accumulation de capitaux, mais au-delà d'un certain temps, on observe des trajectoires stagnantes ou descendantes, notamment chez les individus des groupes 1 et 2. Ces observations seraient à systématiser par une enquête utilisant les récits de vie afin d'effectuer une comparaison des trajectoires. 


\section{Références}

Al ArISs, A. (2010). «Modes of engagement: migration, self-initiated expatriation, and career development», Career Development International, Vol.15, n 4 , p. 338-358.

Al ArIss, A.; Syed, J. (2010). «Capital Mobilization of Skilled Migrants: A Relational Perspective», British Journal of Management, Vol.22, n², p. 286-304.

Becker, G. (1964). Human Capital, Chicago, University of Chicago Press.

BJorkman, I.; Barner-Rasmussen, W.; Li, L. (2004). Managing knowledge transfer in MNCs: the impact of headquarters control mechanisms", Journal of International Business Studies, Vol.35, n 5 , p. 443-455.

Bourdieu, P. (1972). Esquisse d'une théorie de la pratique, précédé de Trois études d'ethnologie kabyle, Genève, Droz.

Bourdieu, P. (1979). «Les trois états du capital culturel», Actes de la recherche en sciences sociales, $\mathrm{n}^{\circ} 30$, p. 3-6.

Bourdieu, P. (1980). «Le capital social. Notes provisoires », Actes de la recherche en sciences sociales, $\mathrm{n}^{\circ} 31, \mathrm{p} .2$.

Bourdieu, P.; WACQUANT L. (2000). «La nouvelle vulgate planétaire», Le Monde Diplomatique, mai, p. 6-7.

Cerdin, J.L. (2004). «Les carrières dans un contexte global», Management \& Avenir, Vol.1/2004, ${ }^{\circ} 1$, p. 155-175.

Cerdin, J.l.; Le Pargneux, M. (2009). «Vers une définition multidimensionnelle de la réussite de la mobilité internationale», Management \& Avenir, Vol.2, ${ }^{\circ} 25$, p. 51-74.

Cerdin, J.l.; Le Pargneux, M. (2010). «Career anchors: a comparison between organization assigned and self-initiated expatriates», Thunderbird International Business Review, Vol.52, n 4, p. 287-299.

Chang, Y-Y.; Gong, Y.; Peng, M.W. (2012). «Expatriate Knowledge Transfer, Subsidiary Absorptive Capacity, and Subsidiary Performance», Academy of Management Journal, Vol.55, n4, p. 927-948.

Chivallon, C. (2000). «D un espace appelant forcément les sciences sociales pour le comprendre », dans J Levy et M Lussault (sous la direction de), Logiques de l'espace, esprit des lieux, Paris, Belin, p. 319-333.

Collings, D.g.; Scullion, H.; Morley, M.J. (2007). «Changing patterns of global staffing in the multinational enterprise: Challenges to the conventional expatriate assignment and emerging alternatives », Journal of World Business, Vol.42, $\mathrm{n}^{\circ} 2$, p. $198-213$.

Doherty, N.; Dickmann, M. (2009). «Exposing the symbolic capital of international assignments », International Journal of HR Management, $\mathrm{Vol} .20, \mathrm{n}^{\circ} 2$, p.301-320.

Doherty, N.; Dickmann, M. (2012). «Measuring the return on investment in international assignments: an action research approach », International Journal of HR Management, Vol.23, $\mathrm{n}^{\circ} 16$, p. 3434-3454.

Elias, N.; Scotson, J. (1975). Logiques de l'exclusion. Enquête sociologique au cour des problèmes d'une communauté, Paris, Fayard.

EMBONG, A.R. (2000). «Globalization and transnational class relations: some problems of conceptualization», Third World Quarterly, Vol. 21, n6, p. 989-1000.
Flamant, N. (2005). «Observer, analyser, restituer. Conditions et contradictions de l'enquête ethnologique en entreprise», Terrain, n44, p. 137-152.

FourNIER, J-M. (2008). «Le capital spatial : une forme de capital, un savoir être mobile socialement inégal. L'exemple de l'élite argentine de Punta del Este (Uruguay)», dans R. Séchet, I. Garat et D. Zeneidi (sous la direction de), Espaces en transactions, Rennes, PUR.

Gherardi L.; Pierre, P., (2010). «Mobilités géographiques et écarts de pouvoir au sein de trois entreprises mondialisées. Mobiles, immobiles et «ubiquistes » , Revue européenne des migrations internationales, Vol.26, $\mathrm{n}^{\circ} 1$, p. 161-185.

Girard, V.; Lagumier, J. (2006). «Risques et catastrophes : de l'enquête de terrain à la construction de l'objet», Genèses, Vol.2, n 63 , p. 128-142.

Laurens, S. (2007). «'Pourquoi' et 'comment' poser les 'questions qui fâchent'? Réflexion sur les dilemmes récurrents que posent les entretiens avec des 'imposants'», Genèses, Vol.4, $\mathrm{n}^{\circ} 69$, p. $112-127$.

Insch, G.s.; Mcintyre, N.; NAPIER, N.K. (2008). «The Expatriate Glass Ceiling: The Second Layer of Glass », Journal of Business Ethics, n ${ }^{\circ}$, p. 19-28.

Levy, J. (1994). L'Espace légitime. Sur la dimension géographique de la fonction politique, Paris : Presses de la FNSP.

Levy, J. (2008). L'invention du monde. Paris, Presses de Sciences-Po.

Loudiyi, S.; Angeon, V.; Lardon, S. (2004). «Capital social et développement territorial. Quel impact spatial des relations sociales?». Colloque Espaces et sociétés aujourd`hui. La géographie dans les sciences sociales et dans l>action, Rennes, 21-22/10/2004

Mcnulty, Y.; De Cieri, H.; Hutchings, K. (2009). «Do global firms measure expatriate $\mathrm{R}$ ? An empirical examination of measures, barriers and variables influencing global staffing practices », International Journal of HR Management, Vol.20, $\mathrm{n}^{\circ}$ 6, p. 1309-1326.

Peterson, R.B.; SArgent, J.; Napier, N.K.; Shim, W.S. (1996). «Corporate Expatriate HRM Policies, Internationalization, and Performance in the World's Largest MNCs », Management International Review, Vol.36, p. 215-230.

Pierre, P. (2003). «Mobilité internationale des élites et stratégies de l'identité». Revue Européenne des Migrations Internationales, Vol.19, ${ }^{\circ} 1$, p. 29-49.

Portes, A.; Guarnizo, L.E.; Landolt, P. (1999). «The study of transnationalism: Pitfalls and promise of an emergent research field», Ethnic and Racial Studies, Vol.22, n² 2, p. 217-237.

Robinson, W.; Harris, J. (2000). «Towards a Global Ruling Class? Globalization and the Transnational Capitalist Class », Science and Technology, Vol.64, ${ }^{\circ} 1$, p. 11-54.

Rofe, M.W. (2003). «'I Want to be Global': Theorising the Gentrifying Class as an Emergent Elite Global Community», Urban Studies, Vol. 40, n 12, p. 2511-2526.

Sechet, R.; Veschambre, V.; Aldhuy, J.; Ripoll, F. (2006). «Demain la géographie sociale. Vers une théorie critique et une approche dimensionnelle de l'espace, au sein des sciences sociales », dans R. Sechet, V. Veschambre, J. Aldhuy et F. Ripoll (sous la direction de), Géopoint 2006 : Demain la géographie sociale, Avignon 
SkLaIR, L. (2001). The Transnational Capitalist Class, Oxford, Blackwell.

Sorokin, P. (1927). Social mobility, New York, Harper and Brothers.

Tizon, P. (1996). «Qu>est-ce que le territoire?», dans G. Di Meo (sous la direction de), Les territoires du quotidien, p.17-27. Paris, L'Harmattan.

Veschambre, V. (2006). «Pour une approche dimensionnelle de l'espace...et une géographie sociale de plain-pied avec les autres sciences sociales», dans R. Sechet et V. Veschambre (sous la direction de), Penser et faire de la géographie sociale. Contributions à une épistémologie de la géographie sociale, Rennes, PUR.

VILAÇA, O. (2001). «L'identité des géographes». Sociologies Pratiques, Vol.5, p. 191-203.

WAGNER, A-C. (2007). Les classes sociales dans la mondialisation, Paris, La Découverte.

Zikic, J.; Bonache, J.; Cerdin, J.L. (2010). «Crossing national boundaries: A typology of qualified immigrants' career orientations », Journal of Organizational Behavior, Vol.31, n5, p. 667-686. 